\title{
The Demand for HIVIAIDS Testing in Women in Zimbabwe
}

Dhoro Netsai L.

\author{
Great Zimbabwe University, Economics Department, Box 1235, Masvingo, Zimbabwe
}

Email: nldhoro@gmail.com

\section{Doi:10.5901/mjss.2014.v5n23p2380}

\begin{abstract}
HIVIAIDS has an adverse socio-economic impact on a nation if not properly managed. One of the ways to prevent the spread of the HIV virus is early HIV testing. This study uses the probit model to explore the factors affecting the woman's decision to get tested for HIV in Zimbabwe. The probit model results show that relational-level and individual-level variables determine HIV testing in women in Zimbabwe. For policy makers concerned about how to encourage early HIV testing among women, policies aimed at providing higher levels of education for women and empowering women in decision making will increase their probability of being tested and would substantially increase early HIVIAIDS testing in Zimbabwe.
\end{abstract}

Keywords: HIV, AIDS, voluntary counseling and testing

\section{Introduction and Background}

Ever since HIVIAIDS was first diagnosed it has undermined progress made in achieving Millennium Development Goals (MDGs), particularly in developing nations (UNAIDS, 2011). According to UNAIDS, Sub-Saharan Africa (SSA) is the worst affected region where HIVIAIDS threatens development, social cohesion, political stability, food security and life expectancy. It further asserts that about $68 \%$ of the people who are HIV infected live in SSA, a region with only $12 \%$ of the global population. Furthermore, about $90 \%$ of the countries that fall under the United Nations Classification of 'generalised epidemic' are located in SSA ${ }^{1}$. HIVIAIDS has also become the leading cause of death in SSA, with approximately 1.2 million people dying from of AIDS-related illnesses annually (UNAIDS, 2012). Although the prevalence of HIV is high in SSA it important to acknowledge that there are wide variations in the infection rates across countries in the region.

In Zimbabwe, since 1985 when the HIV virus was first detected, HIVIAIDS has continued to be a major public health problem and by 2012 approximately 1.6 million Zimbabweans were living with HIVIAIDS, converting to an adult prevalence rate of $14.7 \%$ (Zimstats, 2012). Inspite of a decline in the prevalence rate from $20.1 \%$ in 2005 to $14.26 \%$ in 2009, the country continues to be amongst the 9 countries in the world with the highest HIV infections rates: Swaziland (25.3\%), Botswana (24.8\%), Lesotho (23.6\%), South Africa (17.8\%), Zimbabwe (14.7\%), Zambia (13.1\%), Mozambique (11.5\%) and Malawi (11\%) (Asiedu et al, 2012). The decline in Zimbabwe's HIV prevalence rate was a result of the implementation of interventions around social and behaviour change, increased condom promotion and distribution, coupled with intensified awareness on correct and consistent use of condoms, voluntary male circumcision, HIV testing and counselling, and prevention and control of sexually transmitted infections (ZNASP 2011-2015). According to the Zimbabwe Demographic Health Survey (ZDHS 2010-11) the HIV prevalence rate continues to be higher for women aged 15-24 years than for men of the same age group, 18\% women are HIV positive compared with $12 \%$ for men. This data clearly demonstrate the need for investigating the determinants of HIV testing among women in Zimbabwe. Consequently this paper seeks to investigate the determinants of the demand for HIVIAIDS testing in woman in Zimbabwe.

\section{Literature Review}

HIV counseling and testing has been prioritised as one of the key strategies of preventing and reducing the spread of the

\footnotetext{
1 The United Nations uses three categories to describe the state of the epidemic: low level, concentrated, and generalised. The categories are based on a numerical proxy: a low-level epidemic implies that HIV prevalence has not consistently exceeded $5 \%$ in any defined sub-population, concentrated epidemic means that HIV prevalence is consistently over $5 \%$ in at least one defined sub-population and is below $1 \%$ in pregnant women in urban areas, generalised epidemic implies that HIV prevalence is consistently over $1 \%$ in pregnant women(UNAIDS,2000)
} 
HIVIAIDS epidemic and care globally (Glick, 2005). HIV counseling and testing informs individuals of their HIV serostatus, educates individuals on specific risk reduction practices, provides strategies for partner disclosure and testing, helps patients identify and overcome barriers to risk reductions and links HIV-infected individuals to available medical care (Wanyenze et al., 2006). Based on the evidence from such strong advocacy of this strategy by various stakeholders especially the developmental agencies, the Zimbabwean Government initiated the Voluntary Counseling and Testing Program in 1999. Voluntary Counseling and Testing (VCT) in Zimbabwe typically consists of a pre-test counseling session with a trained counsellor, the sero-test itself, and a post-test session in which individuals are counselled on behaviours to ensure they remain uninfected (if they test negative) or avoid infecting others (if positive). Those testing positive are also provided with emotional support and also directed to services that provide palliative care and other forms of support.

The HIV counseling and testing program was initiated under the New Start brand name in 1999 as a response to the HIV pandemic by the Zimbabwean Ministry of Health and Child Welfare (MoHCW), Population Services International (PSI) and Family Health International (HFI), with funding from the United States Agency for International Development (USAID). Most New Start voluntary, counseling and testing centres are integrated into the existing health service-delivery institutions, such as public clinics and hospitals ${ }^{2}$, nongovernmental organizations, and private health facilities. Between 2005 and 2010 the total number of health facilities offering HIV testing and counselling increased from 395 to 1218 with 1.8 million people getting tested in 2011 (Zimstats, 2012).

Research shows that the implementation of community based VCTs can significantly increase the number of people accessing treatment for the first time (Sweat, 2011; Lachaud, 2007; Glick, 2005). Sweat (2011) conducted a study of communities with access to both community based and standard clinic based VCT and found that more than half of individuals testing for HIV had never been tested before, compared with only 5 percent of those being tested in communities with only standard clinic based VCT. This suggests that community based VCT is a viable option for increasing HIV testing in areas where uptake of testing is low.

\subsection{Importance of HIV Counseling and Testing In Combating HIVIAIDS}

It is difficult to determine whether a person is infected with the HIV virus unless tested. Most individuals go for testing when severe symptoms of the disease are already manifesting themselves and at this stage the CD4 cell ${ }^{3}$ count would be dangerously low. Early testing enables those who test positive to acquire effective antiretroviral treatment therapy (ART) early. Consequently, they would live longer than those who get tested late when their HIV plasma viral load is very high. This is because, when an individual becomes infected with the HIV virus, the virus starts to replicate. The ongoing viral replication drives the disease process and leads to immunological suppression; hence the target for ART is to reduce this viral replication and this can be achieved through early HIV testing.

VCT programs have demonstrated their ability to increase safe sexual behaviour and use of care and support services among adults (Coates et al., 1998). By helping clients learn of their HIV status, VCT provide information and necessary support to change risky behaviours that could lead to HIV infection or transmission (CDC, 1994). In primary health care VCT is regarded as a cost effective way to prevent HIV infection and offers a direct entry point for more effective TB prevention and care for HIV seropositive and seronegative people. Hence promotion of VCT for HIV provides an opportunity to offer interventions for HIV prevention and care.

VCT aids the government in policy making by estimating of the number of people living with HIVIAIDS which enables the government to determine how much of its health budget to allocate to HIVIAIDS. The government will also be in a position to determine how much of its non- financial resources to allocate to HIV response.

HIV testing in women helps in the prevention of mother to child transmission (MTCT), which is the second highest

\footnotetext{
2 The policy on HIV testing in Zimbabwe states that HIV testing is provided within the context of voluntary testing and counseling diagnostic testing (preventing mother-to-child transmission, opportunistic infections and antiretroviral therapy) and blood safety. Rapid tests are most frequently used, and other tests are used for quality assurance. In early 2009, another initiative called PITC provider initiated testing and counseling has been introduced in most public hospitals and clinics in order to convince people to get tested when they come to seek medical care.

${ }^{3}$ CD4 Cell: Also known as "T4" or "helper T cell," the CD4 cell is the primary target of HIV. It performs critical functions such as signalling other parts of the immune system to respond to an infection. Treatment decisions are often based on Viral Load and CD4 count. Normal counts range from 500 to 1500 and in the case of an HIV infection there is a sharp drop in the CD4 count and then the count levels off to around 500-600 cells. In accordance with recent WHO (2003c) guidelines, the decision to start ARV therapy is based on an individual's T-cell (CD4) count. Asymptomatic HIV-positive persons with sufficiently low CD4 counts (below 200 cells/mm3) are also advised to start ARV treatment
} 
mode of HIV transmission. A vast majority of children in Sub-Saharan Africa acquire the HIV virus as a result of MTCT. This normally occurs during child birth, delivery and breast feeding. According to the World Bank (1999), the risk of a baby acquiring the virus from an infected mother ranges from $25 \%$ to $35 \%$ in developing countries in the absence of preventive measures. However, UNAIDS (2011) shows that recent research advances have led to the development of a relatively, inexpensive and logistically feasible antiretroviral (ARV) drug treatment for developing nations that reduces the risk of MTCT by $37 \%$. This procedure involves HIV positive women beginning mother to child prevention treatment immediately before delivery and a one-week post treatment for both mother and her new-born. The success of the intervention hinges upon access to early VCT. Thus, VCT is an important component in combating and reversing HIV and AIDS which is one of the MDGs.

In examining socio-demographic determinants of the demand for HIV testing in Zimbabwe, Sambisa (2008) examined effects of AIDS stigma on uptake of HIV testing in Zimbabwe, with particular emphasis on pathways to HIV testing and reasons for not being tested using a multinomial logistic regression model. Results from the study indicated that testing for HIV was higher among women (30\%) than men (22\%). For women, the main reason for being tested for HIV was accepting testing when it was offered (particularly in the context of antenatal care), whereas for men it was volunteering to be tested. AIDS-related stigma and psychological costs were found to be deterrent determinants to HIV testing uptake.

In an exceptional population based survey, Nyblade et al. (2002) investigated the impact of the costs associated with VCT in rural Uganda. VCT services were offered to all individuals who were given the opportunity to choose to receive the VCT service in their homes or at a nearby clinic. This experiment was meant to effectively reduce some costs associated with VCT to zero. By offering home testing and counseling, costs such as the service itself, transportation costs and the risks to confidentiality associated with using a public facility are eliminated. Despite this significant outreach, the level of demand for the program was low with only $32 \%$ of the women and $35 \%$ of the men having agreed to get tested and receive their results. Nyblade et al.'s (2002) analysis managed to classify the major costs associated with an HIV test into the following categories; financial (includes cost of the test itself and transportation cost) and psychological costs (such as stigma).

Thornton (2005) designed and implemented a randomized experiment in rural Malawi to evaluate the demand for learning HIV results and to estimate subsequent change in behaviour. In this experiment, Thornton used exogenously assigned distance and monetary incentives in order to test their impact on the willingness to learn HIV results after testing. The research found out that distance had a significant impact on VCT centre attendance, with those living far away from the centre being less likely to learn of their HIV status. Thornton also found that monetary incentives also affected the demand for HIV testing as those receiving positive valued incentives were on average twice more likely to learn their HIV status than individuals receiving no incentives.

Nyamathi et al. (2000) found out that increased drug use, unprotected sexual conduct and other high risk behaviour led to higher HIV testing among women. Fernyak et al. (2002), basing on evidence from San Francisco, managed to replicate the same result and also concluded that demand for HIV testing varied according to risk group, race and ethnicity.

In investigating the problem of low HIV testing and disclosure of test results Maman et al. (2001) conducted a study called the Tuelewane project among women at the Muhimbili Health Information Centre (MHIC), in Dar es Salaam, Tanzania. Thematic data analysis revealed that generally a combination of individual and relational level factors influenced individual's decision to test for HIV and to share test results with partners. Perceived benefits of HIV testing and perceptions of risk for HIV were two of the most prominent individual-level variables that influenced VCT clients' decision to be tested. The perception of personal risk of HIV was the major factor driving women to overcome barriers to HIV testing. Women's decision to test for HIV was primarily motivated by having lost a small child or the husband having been sick for a long time. The most salient relational level barriers to HIV testing and status disclosure described by women include fear of partner's reaction, decision making and communication patterns between partners and partner's attitude towards HIV testing.

Thornton (2005) found social ties to be important in determining on HIV testing for women. The strong impact of neighbours on women's attendance to the results centres may be due to additional support received from close neighbours: these are likely to be the next of kin, reducing external or internal psychological costs of attending.

Galvan et al. (2000) analysing the impact of racial and age differences on HIV testing in America found that young people and Afro-Americans were less likely to return for results of their HIV tests. Glick and Sahn (2005) show that individuals with lower levels of education and lower wealth indices are much less likely to be able to identify methods for prevention of HIVIAIDS. This outcome is also confirmed by Michelo et al. (2006) for the case of Zambia, in recent years, marked declines in HIV prevalence in higher educated young people were noticed. 
The current research makes use of some of the variables identified by the different scholars in empirical literature to investigate the determinants the demand for HIVIAIDS in women in Zimbabwe.

\section{Methodology}

The objective of the research is to estimate the determinants of demand for HIV testing in women in Zimbabwe. In this case an individual has either been tested for HIV or not tested; hence the dependent variable in question is a binary variable, taking values for either one (if an individual has been tested) or zero (if not tested). In this case it is mechanically possible to use Ordinary Least Squares estimation procedure yielding the linear probability model (LPM) in which the response probability is linear in the parameters ${ }^{\beta_{j}}$ measures the change in probability of success when ${ }^{X_{j}}$ changes holding other factors fixed.

Such a regression model allows the research to estimate the effects of various explanatory variables on a qualitative event such as HIV testing but such estimation is likely to face statistical problems such as no normality of the error term, $\left(U_{i}\right)$, heteroscedasticity of $U_{i}$, possibility of $Y_{i}$ lying outside the $0-1$ range and the partial effect of any explanatory variable (appearing in level form) is constant and the generally $R^{2}$ lower values (Gujarati 1995, Wooldridge 2004).

In order to avoid these statistical problems, binary response models adopted have the following features: as $X_{i}$ increases, $P=E(Y=1 / X)$ increases but never steps outside the zero-one interval, and the relationship between ${ }^{P_{i}}$ and $X_{i}$ is non-linear (i.e. it approaches zero at a slower and slower rate as ${ }^{X_{i}}$ gets small and approaches one at slower and slower rate as $X_{i}$ gets very large). These models can be estimated using the maximum likelihood technique.

Following Wooldridge (2004), such a model can be stated in the following form:

$$
P(Y=1 / X)=G\left(\beta_{0}+\beta_{1} X_{1}+\ldots . .+\beta_{k} X_{k}\right)=G\left(\beta_{0}+x \beta\right)
$$

Where $x$ denotes a full set of explanatory variables and is a function taking on values strictly between zero and one: $0 \prec G(z) \prec 1$ for all real numbers $z$. The function $G$ can take various non-linear functions. $G$ can follow a logistic function (yielding to the Logit model) or can follow a standard normal cumulative distribution function, resulting in a Probit model.

By using both models, one can come up with the same results since the direction of effect is always the same. However economists tend to favour the normality assumption for $e$, which is why the probit model is more popular than logit in econometrics. In addition, several specification problems are most easily analysed using probit because of properties of the normal distribution (Wooldridge, 2004; p532). Guided by this, this research adopted a probit model as a tool for analysis.

The Probit model used is as follows:

$G(z)=\phi(z) \equiv \int_{-\infty}^{z} \phi(v) d v$

Where $\phi(z)$ is the standard normal density

$\phi(z)=(2 \pi)^{-1 / 2} \exp \left(-z^{2} / 2\right)^{2}$

We rely on calculus in order to find the partial effect of roughly continuous variables on the response probability, if

$X_{i}$ is a roughly continuous variable, its partial effect on $p(x)=P(Y=1 / x)$ is obtained from the following partial derivative: $\frac{\partial p(x)}{d\left(x_{i}\right)}=g\left(B_{0}+x \beta\right) \beta_{j}$ where $g(z) \equiv \frac{\partial G}{\partial z}(z)$

The probit parameters are estimated using the method of maximum likelihood estimation (MLE). The Maximum likelihood (ML) estimation is widely used in health economics, particularly for nonlinear models involving qualitative or limited dependent variables. The Maximum Likelihood function has desirable properties, such as consistency and asymptotic normality, but these rely on the model being fully and correctly specified.

\subsection{Data and Variables}

For the model estimation, data from the 2011-12 Zimbabwe Demographic and Health Survey (ZDHS) was used. This data include information from a nationally representative sample of women age 15-49 years and men age 15-54 years. The ZDHS survey collects information on demographic and health indicators, including social and demographic 
characteristics; marriage and sexual activity; family planning knowledge and use; and HIVIAIDS-related knowledge, attitudes, and behaviour. The ZDHS data also includes self-reported information on AIDS stigma, risk awareness, sexual history and behaviour, HIV testing, and reasons for not being tested for HIV.

A two-stage stratified cluster sampling technique was used to collect the data. In the first stage, a total of 406 primary sampling units or enumeration areas (EAs) were systematically sampled. The EAs were derived from the Zimbabwe Master Sample developed by Zimbabwe's Central Statistical Office after the 2002 population census. In the second stage, a fixed number of households were randomly selected in each primary sampling unit. A total of 9756 households were successfully interviewed, representing a household response rate of 96 percent. In the interviewed households, 9831 eligible women were identified and 9171 completed the interview indicating a 93 percent response rate (Central Statistical Office Zimbabwe 2013).

\subsection{Description of the variables}

The dependant variable HIV testing (EVETEST) is a dummy variable and takes on the value of one if the respondent has been tested for HIV and zero otherwise. Explanatory variables being tested in this study include; age(AGE), wealth(WEALTH), marital status (MSTATUS), place of residence (PLACE), religion(RELIG), individual's level of education (EDU), final say on decision to seek healthcare (DECISION) and partner's education level (PEDULEVEL).

\subsubsection{Age}

Fylkesnes and Siziya (2004) find an inverted-U relationship between age and HIV testing. To analyse whether this relationship holds this study, the study include age in years age and $\mathrm{age}^{2}$ as years as explanatory variables in the study regression. The age variable measures age of respondent in years. The sample was restricted to the range of 15 to 49 years. Current age in completed years was calculated from the century month code of the date of birth of the respondent and the century month code of the date of interview. If the respondent correctly reported her age at her last birthday (and not her age at her next birthday) then the calculated age was rounded up from the reported age, to avoid inconsistencies between the age and the century month code for the birth.

\subsubsection{Education}

Iyaniwura and Oloyede (2006), Jereni and Muula (2008), found a positive and significant association between higher uptakes of VCT with higher levels of education. In this study individual education variable represents the highest level of formal education attained. This is a standardized variable providing level of education in the following categories; no education, primary, secondary and higher education. These categories were corded as follows: no education = 0 ; primary education $=1$; secondary education $=2$ and higher education $=3$.

\subsubsection{Religion}

The results from the study of Namazzi (2010) predict that religion influences personal decision to go for HIV testing. This study therefore includes religion as one of the variables explaining HIV testing among women in Zimbabwe. Religion is hereby coded into the following groups; those affiliated to the traditional religion are assigned the value of zero; Muslim=1; Roman Catholic=2; protestant=3; Pentecostal=4 and other Christians= 5.

\subsubsection{Place of residence}

Namazzi (2010) indicate that the individual's place of residence influences one's decision to undertake HIV testing. The place of residence where the respondent was interviewed was categorized as either urban or rural. This was not the respondent's categorization, but was created based on the cluster or sample point number. When recoding, urban was given the value of one and rural was assigned to the value of zero.

\subsubsection{Wealth}

Individual's wealth status has generally been believed to be a major determinant of health care utilisation in the field of health economics (Lachaud, 2007). The household wealth was estimated using the wealth index and was used to proxy 
the individual's wealth status. Household wealth was grouped into 5 categories: poorest, poor, middle, richer, and richest status, based on an assets-based wealth index quintiles. This wealth variable was recorded in ascending order, with poorest taking the value of zero and the household with the richest status being assigned the value of five.

\subsubsection{Marital Status}

Marital status has influences one's decision to go for an HIV test (Iyaniwura \& Oloyede 2006). In Zimbabwe partners are encouraged to go for an HIV test before they get married. From the survey, all women who were never married are assigned the value of zero, while those who have ever been married are assigned the value of one.

\subsubsection{Final decision making}

This relational-level variable captures the impact of decision making in a household has on HIV testing. According to Maman et al. (2001), women who seek permission prior to HIV testing were found less likely to go to the testing centre. The value of one is assigned to women who make their own health care decisions. The value of zero is assigned to women from households where someone else (e.g. the father or the husband) has the final decision on health care.

\subsubsection{Partner's educational level}

It is believed that a more educated partner is more likely to understand the benefits of HIV testing (Maman et al., 2001). Through lack of understanding, a partner may view the initiative by the other partner to get tested as a sign of infidelity and lack of trust. Partner's education level has been grouped into four stages; no education, primary, secondary and higher education. This has been coded into the following: higher=3, secondary $=2$, primary $=1$, no education and those who do not know their level=0.

\section{Findings and Discussion}

This section presents a summary of the results of the empirical investigation. For the sake of brevity, only a summary of major findings are presented.

The following equation was estimated based on the model described above:

$P(E V E T E S T=1 \mid X)=F\left(\alpha_{i}+\beta_{1} A G E+\beta_{2} A G E^{2}+\beta_{3} E D U+\beta_{4} R E L I G+\beta_{5} P L A C E+\beta_{6} W E A L T H\right.$

$+\beta_{7}$ MSTATUS $+\beta_{8} D E C I S I O N+\beta_{9}$ PEDULEVEL $)+\varepsilon$

Table 1: Probit Model Regression Results

\begin{tabular}{|c|c|c|c|c|}
\hline Evetest & Coef. & Std Err & $z$ & $P \succ|z|$ \\
\hline Cons & -2.942777 & 0.1898721 & -15.50 & 0.000 \\
\hline Age & 0.1042482 & 0.0135421 & 7.70 & $0.000^{\star \star}$ \\
\hline Age 2 & 0.001862 & 0.0002167 & -8.59 & $0.000^{\star \star}$ \\
\hline Edu & 0.1812274 & 0.31685 & 5.72 & $0.000^{\star \star}$ \\
\hline Relig & 0.0138818 & 0.007272 & 1.91 & $0.056^{\star}$ \\
\hline Place & -0.0219467 & 0.526826 & -0.42 & 0.677 \\
\hline Wealth & 0.0642526 & 0.0237153 & 2.71 & $0.007^{\star \star}$ \\
\hline Mstatus & 0.2262232 & 0.609108 & 3.71 & $0.000^{\star \star}$ \\
\hline Decision & 0.0983165 & 0.0353371 & 2.78 & $0.005^{\star \star}$ \\
\hline Pedulevel & 0.1542655 & 0.025549 & 6.04 & $0.000^{\star \star}$ \\
\hline
\end{tabular}

** Significant at 1\% *Significant at 10\%

Number of obs $=9171 \mathrm{LR} \mathrm{chi}^{2}(16)=932.34 \mathrm{Prob}>\mathrm{chi}^{2}=0.0000$

Log likelihood $=-4595.9328$ Pseudo $R^{2}=0.0921$ 
Table 2: Predicted Probabilities

\begin{tabular}{|c|c|}
\hline Mstatus & Prediction \\
\hline 0 & 0.1874 \\
\hline 1 & 0.2542 \\
\hline Religion & Prediction \\
\hline 0 & 0.2218 \\
\hline 1 & 0.2301 \\
\hline 2 & 0.2344 \\
\hline 3 & 0.2387 \\
\hline 4 & 0.2430 \\
\hline 5 & 0.2473 \\
\hline Place & Prediction \\
\hline 0 & 0.2348 \\
\hline 1 & 0.2281 \\
\hline Wealth & Prediction \\
\hline 0 & 0.2070 \\
\hline 1 & 0.2259 \\
\hline 2 & 0.2457 \\
\hline 3 & 0.2663 \\
\hline Decision & Prediction \\
\hline 0 & 0.2219 \\
\hline 1 & 0.2522 \\
\hline Edu & Prediction \\
\hline 0 & 0.1532 \\
\hline 1 & 0.2000 \\
\hline Pedu & Prediction \\
\hline 0 & 0.1800 \\
\hline 1 & 0.2232 \\
\hline 2 & 0.2719 \\
\hline 3 & 0.3254 \\
\hline
\end{tabular}

The variable age is statistically significant at $1 \%$ level of significance. Demand for HIV testing is flexible with age. The coefficient for age variable is positive implying that the probability of getting tested increases with age. Age $^{2}$ has a negative coefficient, showing that although demand increases with age, it will fall as people get older. This supports the findings by Galvan et al. (2000) who found the variable age to be significant in explaining HIV testing.

The estimated coefficient for education is positive and significantly different from zero at $1 \%$ level of significance. This shows that women with a higher level of education are more likely to go for HIV testing as compared to those with lower level of education, confirming literature findings (Walque, 2006; Hargreaves and Glynn, 2002). The predicted probability of being tested is 0.3159 for women with higher educational level compared to women with no education have a probability of only 0.1532 . These results signify that education has a huge impact on HIV prevention probably because such women may have more understanding of the benefits of such services and are more likely to respond to health promotion messages.

Religion is a significant predictor of HIV testing in the study. However the level of influence differs across different religion beliefs as Pentecostals are estimated to have a 0.3252 probability of getting tested unlike those who are affiliated to the traditional religion with a probability of 0.2778 (Namazzi,2010).

The coefficient for the place variable is negative and statistically insignificant. The variable does not help in explaining the demand for HIV testing by women in Zimbabwe. The explanation for the negative coefficient might be that in Zimbabwe there are no well-established social networks that can be used as a source of support in the event that one is found to be HIV positive. This might be attributed to the high level of stigmatisation still found in the Zimbabwean society. This will therefore result in women being less likely to go for HIV testing.

In conformity to theory (Namazzi,2010), the coefficient of the wealth variable is positive and statistically significant from zero at $1 \%$ level of significance suggesting that, conditional on all covariates, women from rich households have a higher likelihood of up taking an HIV test than those from poor households. Women who come from richest households are more likely to get tested; this group has a probability of 0.2663 of getting tested. Women from poorest households are 
less likely to be tested with a probability of 0.2070 . This outcome is in line with the Grossman's model of health economics model that theorise a positive relationship between the demand for health care and wealth.

The estimated coefficient for marital status variable is significant at $1 \%$. This indicates that married women are more likely to be tested than non -married women with the probability of married women being tested being higher (0.2542) compared to that for non-married women (0.1874). The results are in line with the findings of lyaniwura and Oloede (2006) on the influence of marital status on the decision to go for an HIV test.

The coefficient relational-level variable final decision on health care is positive and significant at $1 \%$ level of significance. Women who make their own health care decisions are more likely to go for HIV testing than women who do not have a final say when it comes to their own health care decisions. Women who make their own health care decisions have a probability of 0.2522 of getting tested for HIV. Those who do not make their own decisions have a probability of only 0.2219 of getting tested. These results indicate that women who depend on their partners' approval to decide on testing are limited in their use of VCT services. These results are in line with those found by Maman et al, 2001 in Kenya where the fear of their partner's reaction was cited as a factor that limited testing by women.

The coefficient of the partner's education level variable is positive and significant at $1 \%$ level of significance. Women who have partners that have a higher level of education are more likely to get tested than those whose partners have less or no education at all. This result conforms to Michelo et al, 2006 findings and indicates that education patterns of partners are critically important in women's decision to get tested for HIV. Women with partners who have no education have a probability of only 0.1800 of getting tested. Women with partners who have reached higher education on the other hand have a probability of 0.3254 .

\section{Conclusion}

The objective of the study was to investigate the determinants of the demand for HIV testing in women in Zimbabwe. Relational-level and individual-level variables were found to be the main predictors of HIV testing in women. Women's demand for HIV testing was found to be influenced by relational-level variables such as decision making patterns in the household and partner's educational level. Individual level factors such as; marital status, individual education level, wealth, age and religion were also found to be predictors of HIV testing in women. The analysis made in this study is important for policy purposes because it establishes the predictors of HIV testing for women, either within or beyond the control of policy makers. For policy makers concerned about how to encourage early HIV testing among women, policies aimed at providing higher levels of education for women will increase their probability of being tested. Besides formal education, more educational campaigns need to be instituted to educate women about the benefits of going for early HIV testing. Awareness also needs to be carried out on how certain peoples' behaviours can put them at high risk. It is also essential to empower women in decision making when it comes to health care. However there is also need for further studies to analyse the determinants of HIV testing for women considering different behaviours as dictated by location such as the different behaviours found between rural and urban environments.

\section{References}

Asiedu, C., Asiedu, E., \& Owusu, F. (2012) The Socio-Economic Determinants of HIV/AIDS Infection Rates in Lesotho, Malawi, Swaziland and Zimbabwe, Journal of Development Policy Review 30(3): 305-326.

Centre for Disease Control and Prevention (1994). HIV Counseling, Testing, and Referral Standards and Guidelines. Atlanta, GA: U.S. Department of Health and Human Services.

Coates, T. (1998). Voluntary HIV Counseling and Testing (VCT) Reduces Risk Behaviour in Developing Countries: Results from the Voluntary Counseling and Testing study, Paper presented at the International Conference on AIDS, Geneva, Switzerland, 28 June -3 July.

D. Friesner et al. (2007). Determinants of Demand for HIV Testing: Evidence from California Outpatients Clinics. Californian Journal of Health Promotion 2007, Volume 5, Issue 4, 52-75.

De Walque, D. (2006). "Who gets AIDS and how? The determinants of HIV infection and sexual behaviours in Burkina Faso, Cameroon, Ghana, Kenya and Tanzania," Policy Research Working Paper 3844. Washington, DC: World Bank.

Demographic and Health Surveys, (2010/2011). Zimbabwe, DHS: 183-234.

DeZoysa, I. Phillips, K. A., Kamenga, M.C., O'reilley, K., Sweat, M., White, R., Grinstead, O.A. \& Coates, T. (1995). Role of HIV Counseling and Testing in Changing Risk Behaviour in Developing Countries. AIDS, 9(Suppl. A), S95-S101.

Duflo, E. (2006). Field Experiments in Development Economics. Department of Economics and Abdul Latif Jameel Poverty Action Lab, Massachusetts Institute of Technology, BREAD, CEPR, NBER.

Fan, J. (1992). Design-Adaptive Nonparametric Regression. Journal of the American Statistical Association, 87(420), pp. 998-1004.

Fernyak, S., Page-Shafer, K., Kellog, T., McFarland, W., \& Katz, M. (2002). Risk Behaviours and HIV Incidence Among Repeat Testers 
at Publicly funded HIV testing sites in San Francisco. Journal of AIDS 31: 63-70.

Fylkesnes, K. \& Siziya, S. (2004). A randomized trial on acceptability of voluntary HIV counselling and testing. Tropical Medicine and International Health, 9(5):566-72.

Glick, P. (2005). Scaling Up HIV Voluntary Counseling and Testing In Africa: What Can Evaluation Studies Tell Us About Potential Prevention Impacts. Education Review 29 (4): pp.331-357.

Gujarati, N.D. (2004). Basic Econometrics. (Fourth Edition). The McGraw Hill, New York.

Hargreaves, J. R. \& Glynn. J. R. (2002). Educational Attainment and HIV-1 Infection in Developing Countries: a Systematic Review. Tropical Medicine and International Health 7, 489-498.

Iyaniwura, C. A. \& Oloyede, O. (2006). HIV testing among youths in a Nigerian local population. West Africa Journal of Medicine, 25(1):27-31.

Jereni, B. H. \& Muula, A. S. (2008). Availability of supplies and motivations for accessing voluntary HIV counseling and testing services in Blantyre, Malawi Biomedical Central Health Services Research, 8:17.

Namazzi, J. A. (2010). Determinants of Using Voluntary Counseling and Testing for HIVIAIDS in Kenya, Journal of Management Policy and Practice, Vol. 11, Iss. 5, pp.89- 96

Kellerman, S., Lehman, J. S., \& Lansky, A. (2002) HIV testing within at-risk populations in the United States and the reasons for seeking or avoiding HIV testing. Journal of Acquired Immune Deficiency Syndromes. 31:202-210.

Lachaud, J. (2007). HIV prevalence and poverty in Africa: Micro and Macro-econometric Evidences Applied to Burkina Faso. Journal of Health Economics 26: 483-504

Maman, S., Mbwambo. J., Hogan. N. M., Kilonzo, G. P., \& Sweat, M. (2001). Women's Barriers to HIV-1 Testing and Disclosure: Challenges for HIV-1 Voluntary Counselling and Testing. AIDS Care, 13(5): 595-603.

Mapenzauswa, S. (2004). Zimbabwe Holds First National Meeting on AIDS Crisis. Reuters NewsMedia.

Marcel, Z., Beat E., Neuenschwander, A. J., Francoise D. \& Vlahov, D. (2000). HIV Testing and Retesting for Men and Women in Switzerland. European Journal of Epidemiology, Vol. 16, No. 2 (Feb., 2000), pp. 123-133.

Michelo, C., Sandoy, I. F., \& Fylkesnes, K. (2006). Marked HIV Prevalence Declines in Higher Educated Young People: Evidence from Population-based Surveys (1995-2003) in Zambia. A0, no. 7: 1031-8.

National HIVIAIDS Strategic Framework 2011-2015, Republic of Zimbabwe; 2012.

Nyamathi, A., Stein, J. \& Swanson. (2000). Personal, Cognitive, Behavioural and Demographic Predictors of HIV testing and STDs in homeless women. Journal of Behavioural Medicine. 23, no. 2: 123-147.

Nyblade, L., Gray, R., Makumbi, F., Lutalo, T., Menken, J., Wawer, M., Sewankambo, N. \& Serwadda, D. (2000). HIV Risk Characteristics and Participation in Voluntary Counseling and Test HIV Risk Characteristics and Participation in Voluntary Counseling and Testing in rural Rakai district, Uganda. Paper presented at the $13^{\text {th }}$ International Conference on HIVIAIDS. Durban, South Africa.

Sambisa, W. (2008). AIDS Stigma and Uptake of HIV Testing in Zimbabwe. Demographic Health Survey Working Paper Series.

Sweat, M. et al. (2011), Community-based intervention to increase HIV testing and case detection in people aged 16-32 years in Tanzania, Zimbabwe, and Thailand (NIMH Project Accept, HPTN 043): a randomised study. Lancet Infect Dis 11(7):525-32.

Thornton, R. (2005). The Demand for and Impact of Learning HIV Status: Evidence from a Field Experiment. MIMEO.

UNAIDS, (2002). Zimbabwe Epidemiological Fact Sheets on HIV and AIDS and Sexually Transmitted Infections.2002 Update.

UNAIDS, (2011). UNAIDS Output Report. Geneva: UNAIDS

UNAIDS, (2013) Report on the GLOBAL AIDS EPIDEMIC. Geneva: UNAIDS

Wanyenze, R., Kamya, M., Liechty, C. A., Ronald, A., Guzman, D. J., Wabwire-Mangen, F., et al. (2006). HIV Counseling and Testing Practices at an Urban Hospital in Kampala, Uganda. AIDS and Behaviour, 10, 361-367.

Wooldridge, J. (2004). Introductory econometrics: A modern approach. Cincinnati, OH: South-Western Publishing.

Wortley, P., Chu, S., Diaz, T., Ward, J., Doyle, B. \& Davison, A. (1995). HIV testing patterns: Where, Why and When were persons with AIDS tested for HIV? AIDS 9: 487-492. 